\title{
Preoperative voiding detrusor pressures do not predict stress incontinence surgery outcomes
}

\author{
Anna C. Kirby • Charles W. Nager • \\ Heather J. Litman • Mary P. FitzGerald • \\ Stephen Kraus • Peggy Norton • Larry Sirls • \\ Leslie Rickey • Tracey Wilson • Kimberly J. Dandreo • \\ Jonathan P. Shepherd • Philippe Zimmern • \\ for the Urinary Incontinence Treatment Network
}

Received: 30 September 2010 / Accepted: 15 November 2010/Published online: 10 December 2010

(C) The Author(s) 2010 This article is published with open access at Springerlink.com

\begin{abstract}
Introduction and hypothesis The aim of this study was to determine whether preoperative voiding detrusor pressures were associated with postoperative outcomes after stress incontinence surgery.

Methods Opening detrusor pressure, detrusor pressure at maximum flow ( $p_{\text {det }} \mathrm{Q}_{\max }$ ), and closing detrusor pressure were assessed from 280 valid preoperative urodynamic studies in subjects without advanced prolapse from a multicenter randomized trial comparing Burch and autologous fascia sling procedures. These pressures were compared between subjects with and without overall success, stress-specific success, postoperative detrusor
\end{abstract}

This work was performed at each of the institutions listed below through the Urinary Incontinence Treatment Network.

A. C. Kirby $\cdot$ C. W. Nager $(\bowtie)$

Reproductive Medicine, University of California San Diego,

San Diego, CA, USA

e-mail: cnager@ucsd.edu

H. J. Litman $\cdot$ K. J. Dandreo

New England Research Institute,

Watertown, MA, USA

M. P. FitzGerald

Obstetrics and Gynecology, Loyola University Medical Center,

Maywood, IL, USA

S. Kraus

Urology, University of Texas Health Sciences Center,

San Antonio, TX, USA

P. Norton

Obstetrics and Gynecology, University of Utah,

Salt Lake City, UT, USA overactivity, and postoperative urge incontinence using independent sample $t$ tests.

Results There were no clinically or statistically significant differences in mean preoperative voiding detrusor pressures in any comparison of postoperative outcomes.

Conclusions We found no evidence that preoperative voiding detrusor pressures predict outcomes in women with stress predominant urinary incontinence undergoing Burch or autologous fascial sling procedures.

Keywords Closing detrusor pressure - Opening detrusor pressure - Urinary stress incontinence $\cdot$ Urinary urge incontinence $\cdot$ Urodynamics $\cdot$ Voiding detrusor pressure
L. Sirls

Urology, William Beaumont Hospital,

Royal Oak, MI, USA

L. Rickey

Urology, University of Maryland,

Baltimore, MD, USA

T. Wilson

Urology, University of Alabama at Birmingham,

Birmingham, AL, USA

J. P. Shepherd

Obstetrics, Gynecology, and Reproductive Science,

Magee Women's Hospital, University of Pittsburgh,

Pittsburgh, PA, USA

P. Zimmern

Urology, University of Texas Southwestern,

Dallas, TX, USA 


$\begin{array}{ll}\text { Abbreviations } \\ \text { BMI } & \text { Body mass index } \\ \text { CDP } & \text { Closing detrusor pressure } \\ \mathrm{cm} & \text { Centimeter } \\ \mathrm{CMG} & \text { Cystometrogram } \\ \mathrm{DO} & \text { Detrusor overactivity } \\ \mathrm{H}_{2} \mathrm{O} & \text { Water } \\ \mathrm{kg} & \text { Kilogram } \\ \mathrm{m} & \text { Meter } \\ \mathrm{ml} & \text { Milliliter } \\ \mathrm{MCC} & \text { Maximum cystometric capacity } \\ \text { MESA } & \text { Medical Epidemiological and Social Aspects of } \\ & \text { Aging } \\ \text { OAB } & \text { Overactive bladder } \\ \text { ODP } & \text { Opening detrusor pressure } \\ p_{\text {abd }} & \text { Abdominal pressure } \\ p_{\text {det }} & \text { Detrusor pressure } \\ p_{\text {det }} Q_{\text {max }} & \text { Detrusor pressure at maximum flow } \\ p_{\text {ves }} & \text { Bladder pressure } \\ \text { PFS } & \text { Pressure-flow studies } \\ \text { Postop } & \text { Postoperative } \\ \text { PVR } & \text { Postvoid residual } \\ Q_{\text {max }} & \text { Maximum flow } \\ \text { ROC } & \text { Receiver operator characteristic } \\ \text { SD } & \text { Standard deviation } \\ \text { SISTEr } & \text { Stress Incontinence Surgical Treatment Efficacy } \\ & \text { Trial } \\ \text { SUI } & \text { Stress urinary incontinence } \\ \text { TVT } & \text { Tension-free vaginal tape } \\ \text { UDS } & \text { Urodynamic studies } \\ \text { UITN } & \text { Urinary Incontinence Treatment Network } \\ \text { USI } & \text { Urodynamic stress incontinence } \\ \text { UUI } & \text { Urge urinary incontinence } \\ & \end{array}$

\section{Introduction}

Preoperative urodynamic studies (UDS) are commonly performed prior to stress incontinence (SUI) surgery. If urodynamic measures could predict overall success, stressspecific success, postoperative detrusor activity (DO), or the need for postoperative urge urinary incontinence (UUI) treatment, then these measures might be useful for preoperative counseling.

Recent studies suggest that voiding detrusor pressure values during preoperative UDS predict postoperative outcomes. Digesu et. al. found that postoperative incontinent women after Burch colposuspension had lower preoperative opening detrusor pressures (ODP) and closing detrusor pressures (CDP) than continent women [1]. Similarly, in a study of 35 women with urodynamic mixed incontinence, defined as urodynamic stress incontinence (USI) and DO, investigators found that higher ODP during preoperative urodynamic testing predicted persistent $\mathrm{DO}$ after tension-free vaginal tape (TVT) surgery. ODP was also higher in subjects with postoperative overactive bladder (OAB), but this difference was not statistically significant [2].

We hypothesized that preoperative voiding detrusor pressures would be associated with postoperative outcomes after SUI surgery in women with stress predominant SUI. Specifically, we sought to determine whether ODP, detrusor pressures at maximum flow $\left(p_{\mathrm{det}} Q_{\mathrm{max}}\right)$, or CDP were associated with, or predictive of, overall success, stressspecific success, postoperative DO, and postoperative treatment for UUI. We also evaluated whether preoperative detrusor after-contractions were more common in women who were treated for postoperative UUI.

\section{Materials and methods}

\section{SISTEr trial and subjects}

This study is a secondary analysis of data from the Stress Incontinence Surgical Treatment Efficacy Trial (SISTEr). In the original trial, 655 women were enrolled from February 2002 to June 2004. Details of the SISTEr study methods have been published previously [3] and are briefly outlined here. Inclusion criteria for enrollment included: (1) predominant SUI: stress $>$ urge score on the Medical, Epidemiological and Social Aspects of Aging Questionnaire (MESA) [4], (2) positive stress test (observed leakage from the external urethral meatus coincident with a cough or Valsalva maneuver with a bladder volume $\leq 300 \mathrm{ml}$ ), (3) urethral hypermobility as evidenced by resting or maximum straining Q-tip angle $>30^{\circ}$ [5], (4) maximum cystometric capacity $(\mathrm{MCC}) \geq 200 \mathrm{ml}$, and (5) postvoid residual (PVR) $<150 \mathrm{ml}$. All study procedures were approved by the institutional review board of each participating clinical center and the Biostatistical Coordinating Center with written informed consent obtained from all women prior to enrollment.

Urodynamic studies and quality control

Baseline urodynamic testing (free uroflowmetry, filling cystometry, and pressure-flow studies) were performed on all participants prior to surgery based on a standardized protocol implemented by all 20 urodynamic testers at the nine continence treatment centers. The SISTEr UDS protocol complied with terminology from the Standardization Committee of the International Continence Society [6] and technical recommendations from the Good Urodynamic Practice guidelines [7]. Standardized interpretation guidelines were used by on-site (local) physician reviewers. The details of our specific UDS Protocol and Interpretation Guidelines are available on the Urinary Incontinence 
Treatment Network website at http://www.uitn.net/resources forphysicians.htm. A Urodynamics Working Group supervised all aspects of urodynamic protocol development, procedural performance, and interpretation reliability $[8,9]$.

Pressure-flow studies

After standing filling cystometry, study participants sat to void for pressure-flow studies (PFS). For PFS pressure data to be considered valid, they had to satisfy the following criteria: (1) legible signals, (2) subject voided, (3) bladder pressure $\left(p_{\text {ves }}\right)$ and abdominal pressure $\left(p_{\text {abd }}\right)$ measuring systems properly functioning at PFS baseline and $Q_{\max }$, (4) $p_{\text {det }}$ at PFS baseline greater than $-5 \mathrm{~cm} \mathrm{H}_{2} \mathrm{O},(5) p_{\text {det }}$ at PFS baseline no more than $15 \mathrm{~cm} \mathrm{H}_{2} \mathrm{O}$ greater than $p_{\text {det }}$ at $\mathrm{MCC}$ (insuring that change of position and transducer adjustment did not cause artifact in pressure measurement), (6) prevoid cough spikes demonstrated at least $70 \%$ agreement between the $p_{\text {ves }}$ and $p_{\text {abd }}$ signals (smaller spike at least $70 \%$ of the larger spike) [10] to demonstrate that the bladder and abdominal pressure transducers were functioning well, and (7) the local reviewer found no other reason to invalidate the study. For this analysis to be consistent with our other plausibility criteria, we also excluded all PFS with voiding detrusor pressure measures (ODP, $p_{\operatorname{det}} Q_{\max }$, or CDP) less than $-5 \mathrm{~cm} \mathrm{H}_{2} \mathrm{O}$.

Preoperative voiding detrusor pressure measurements

We followed the methods used by Panayi et al. 2009 [2] to determine ODP and CDP, defining them as the detrusor pressure $1 \mathrm{~s}$ prior to the onset of flow and $1 \mathrm{~s}$ prior to cessation of flow, respectively, as recorded by the uroflowmeter. For ODP, to evaluate the appropriateness of the 1-s delay for the urine to flow through the urethra, emerge from the patient, and register on the uroflowmeter, we also recorded the highest detrusor pressure in the 1 to $5 \mathrm{~s}$ preceding the onset of flow on the uroflowmeter and how many seconds prior to flow it occurred. In more than $90 \%$ of the signals, the highest detrusor pressure in the 1 to $5 \mathrm{~s}$ preceding the onset of flow was equal to ODP at $1 \mathrm{~s}$ prior to onset of flow, and therefore, we only report ODP at $1 \mathrm{~s}$ for this analysis. Detrusor pressure at maximum flow $\left(p_{\operatorname{det}} Q_{\max }\right)$ was recorded from the $Q_{\max }$ auto-annotation on each UDS signal. The reviewers were blinded to all outcome measures when the urodynamic data were extracted.

After-contractions are sometimes found as a pressure increase after flow ceases at the end of micturition [11]. The significance of this event is not understood, and there is no universally accepted definition. For the purpose of this review, we defined after-contractions as any detrusor pressure within $5 \mathrm{~s}$ of the end of flow greater than any detrusor pressure during flow.
Preoperative voiding detrusor pressure quality control

Fifty arbitrarily selected urodynamic signals were jointly reviewed by the first and second authors for education, training, and clarification of definitions. Subsequently, a statistician (HL) randomly selected 22 signals for independent review to evaluate inter-rater reliability. A priori, $80 \%$ agreement was determined to be acceptable inter-rater reliability, and pressure measures with a numerical difference of $10 \%$ or less were considered the same since $\leq 10 \%$ differences in pressure are seldom clinically important. It was determined a priori that, if acceptable reliability could be established, a single interpreter would perform the remainder of the data extraction.

Overall treatment success and stress-specific success

Success was evaluated at 24 months. Overall treatment success was defined as no self-reported SUI symptoms, a negative provocative standardized $300-\mathrm{ml}$ stress test, no retreatment for SUI, less than $15 \mathrm{~g}$ of urine on a 24-h pad test, and no leakage on a 3-day diary. Stress-specific success was defined as no SUI symptoms, a negative stress test, and no SUI re-treatment [12].

\section{Detrusor overactivity}

DO was defined as a urodynamic observation of involuntary detrusor contractions during the filling phase [6]. Preoperative DO was DO during the baseline UDS and postoperative DO was DO during UDS at the 24-month urodynamic visit. Since preoperative DO is likely to be associated with postoperative $\mathrm{DO}$, in our analysis of postoperative $\mathrm{DO}$, we performed two analyses, one excluding and one including subjects with preoperative DO.

\section{Treatment of postoperative urge incontinence}

Treatment of postoperative UUI was defined as treatment of clinically diagnosed new-onset or persistent UUI after the 6-week follow-up visit with any clinically acceptable treatment for $\mathrm{OAB}$. This parameter was assessed at 3,6, 12,18 , and 24 months.

Preoperative pelvic organ prolapse

Pelvic organ prolapse was assessed during preoperative physical examination using POPQ definitions $[12,13]$. For this secondary analysis, we excluded subjects with pelvic organ prolapse past the introitus (stage 3 or 4) because prolapse could artificially increase pressures by causing urethral obstruction, and exclusion of prolapse subjects is consistent with previous literature evaluating the role of preoperative voiding detrusor pressures $[1,2]$. 
Statistics

The distributions of preoperative voiding detrusor pressure measures were assessed for normality. There was no strong evidence of deviation from normality, so independent sample $t$ tests were used to test for differences in detrusor pressures by overall and stress success status, DO status, and UUI treatment status. Based on the number of subjects with and without postoperative UUI, there was at least $80 \%$ power to detect a difference in mean ODP values of $5 \mathrm{~cm} \mathrm{H}_{2} \mathrm{O}$ assuming that mean ODP of subjects with postoperative UUI was 13 with a standard deviation of 10 [14]. Pearson chi-square tests were used to test for relationships between categorical variables (e.g., after-contractions). Analyses were carried out using SAS statistical software Version 9.2 (SAS Institute, Inc., Cary, NC).

\section{Results}

\section{SISTEr trial and subjects}

The 655 study subjects had a mean age of 52 years (standard deviation (SD) 10, range 28 to 81) and a mean BMI of $30 \mathrm{~kg} / \mathrm{m}^{2}\left(\mathrm{SD}, 6 \mathrm{~kg} / \mathrm{m}^{2}\right)$. The ethnic distribution was: $73 \%$ non-Hispanic White, $11 \%$ Hispanic, $7 \%$ nonHispanic Black, and 9\% non-Hispanic Other.

Pressure-flow studies and detrusor pressure quality control

The 655 subjects all underwent preoperative UDS. Seventyeight UDS had invalid or implausible cystometrogram (CMG) studies, and 191 had invalid or implausible PFS as described previously [9]. We excluded an additional 13 studies that were not captured on standardized electronic equipment, one study from which data were not properly extracted, nine studies in which the pressure catheter fell out before $Q_{\max }$, and 46 signals with implausible pressures (less than $\left.-5 \mathrm{~cm} \mathrm{H}_{2} \mathrm{O}\right)$ at ODP $(n=14), p_{\text {det }} Q_{\max }(n=8)$, or CDP $(n=23)$. We subsequently excluded 46 of these 326 high-quality PFS because the subjects had stage 3 or 4 prolapse. This left 280 PFS studies that met our predetermined quality control measures for this analysis.
Evaluation for potential selection bias

An analysis was performed to determine if there was possible selection bias introduced by excluding subjects with invalid or implausible UDS subjects. Table 1 compares the postoperative outcome rates in the 280 subjects with valid UDS who were included in this analysis to the postoperative outcome rates in the 269 subjects that were excluded (549 subjects in SISTEr had stage 2 prolapse or less, and not all subjects had all outcome measures recorded). No differences between those included and excluded were detected.

Inter-rater reliability

Inter-rater agreement was $95 \%$ (21/22) for ODP, $82 \%(18 / 22)$ for $p_{\mathrm{det}} Q_{\max }, 91 \%(20 / 22)$ for CDP, and $91 \%$ (20/22) for the presence or absence of after-contractions. This inter-rater agreement met our reliability criteria, and most of the data extraction was then obtained by a single interpreter (AK).

Preoperative voiding detrusor pressures

Mean and standard deviation (SD) preoperative voiding detrusor pressures for all 280 studies were ODP 13.5 (9.7), $p_{\text {det }} Q_{\max } 19.8$ (11.3), and CDP $18.3(15.8) \mathrm{cm} \mathrm{H}_{2} \mathrm{O}$.

Overall treatment success and stress-specific success

Table 2 shows the mean preoperative voiding detrusor pressures in subjects with overall success and failure and stress-specific success and failure. There were no significant differences in mean ODP, $p_{\text {det }} Q_{\max }$, or CDP between the success and failure groups.

Postoperative detrusor overactivity

In SISTEr, 9.3\% (60/645) of subjects had preoperative DO and $8.9 \%(45 / 506)$ had postoperative DO. Table 3 presents mean preoperative voiding detrusor pressures grouped by postoperative DO status in subjects stratified by the presence or absence of preoperative DO. In subjects with preoperative USI or preoperative urodynamic mixed incontinence (USI and DO), there was no significant difference
Table 1 Postoperative outcome rates for included and excluded subjects

$D O$ detrusor overactivity, UUI urinary urge incontinence

\begin{tabular}{lccc}
\hline & Included subjects & Excluded subjects & $p$ value \\
\hline Overall success & $38.5 \%(85 / 221)$ & $35.1 \%(74 / 211)$ & 0.47 \\
Stress-specific success & $52.9 \%(120 / 227)$ & $57.3 \%(129 / 225)$ & 0.34 \\
Postoperative DO & $12 \%(25 / 207)$ & $7.5 \%(16 / 214)$ & 0.11 \\
Treatment for postoperative UUI & $19.6 \%(55 / 280)$ & $22.7 \%(61 / 269)$ & 0.38 \\
\hline
\end{tabular}


Table 2 Preoperative voiding detrusor pressures in success and failure groups

\begin{tabular}{llllll}
\hline & $\begin{array}{l}\text { Overall success } \\
(n=85)\end{array}$ & $\begin{array}{l}\text { Overall failure } \\
(n=136)\end{array}$ & $p$ value & $\begin{array}{l}\text { Stress-specific } \\
\text { success }(n=120)\end{array}$ & $\begin{array}{l}\text { Stress-specific } \\
\text { failure }(n=107)\end{array}$ \\
\hline ODP & $11.3(9.5)$ & $13.6(8.6)$ & 0.07 & $12.3(9.6)$ & $13.6(8.9)$ \\
$p_{\operatorname{det}} Q_{\max }$ & $19.1(11.8)$ & $19.7(11.2)$ & 0.70 & $19.8(11.5)$ & $19.4(11.4)$ \\
CDP & $16.0(12.9)$ & $19.0(15.7)$ & 0.14 & $16.8(13.0)$ & $19.1(16.4)$ \\
\hline
\end{tabular}

Values are reported as mean in centimeter $\mathrm{H}_{2} \mathrm{O}(\mathrm{SD})$

$O D P$ opening detrusor pressure, $p_{d e t} Q_{\max }$ detrusor pressure at maximum flow, $C D P$ closing detrusor pressure

in voiding detrusor pressures in subjects with and without postoperative DO.

Treatment for postoperative urge incontinence

Of the 55 subjects treated for postoperative UUI in this study, all 55 were treated with medications, 11 were also treated with biofeedback or behavioral training, and four were treated with other therapies. Table 4 shows the mean preoperative voiding detrusor pressures for subjects who were and were not treated for postoperative UUI. No preoperative voiding detrusor pressures were statistically different.

\section{After-contractions}

Twenty-six percent (8/31) of subjects with after-contractions received treatment for postoperative UUI compared to $19 \%$ $(47 / 249)$ of those without after-contractions. This rate difference was not statistically different $(p=0.36)$.

\section{Discussion}

In this analysis of preoperative urodynamic data from a large stress incontinence surgery trial, we found no voiding detrusor pressure parameter that was associated with overall success, stress-specific success, DO, or treatment for UUI after surgery. This implies that none of these measures could be used clinically to predict which patients would have these postoperative outcomes.
There was no difference in the prevalence of aftercontractions when we compared those who received treatment for postoperative UUI to those who did not, leaving the significance of this finding still not understood [11].

We acknowledge that lack of significance of superiority tests does not necessarily imply lack of difference. However, this study had an adequate sample size $(n=280)$ to detect large, clinically relevant differences, so it is unlikely that low power was the reason for not detecting differences in preoperative voiding detrusor pressures by success, DO, or UUI treatment. For instance, based on the number of subjects with and without postoperative UUI ( $n=225$ vs $n=$ 55 ), there was at least $80 \%$ power to detect a difference in mean ODP values of $5 \mathrm{~cm} \mathrm{H}_{2} \mathrm{O}$ assuming that mean ODP of subjects with postoperative UUI was 13 with a standard deviation of 10 [14].

Unlike previous investigators who found that subjects with postoperative continence had ODP values $9 \mathrm{~cm} \mathrm{H}_{2} 0$ higher than those with postoperative incontinence, [1] we found that our subjects with postoperative continence had ODP values 1 to $2 \mathrm{~cm} \mathrm{H}_{2} \mathrm{O}$ lower than those with postoperative incontinence. Our studies were similar, and both had over 200 subjects, but they differ in the following ways: (1) our study included Burch and sling and their study had Burch procedures only, (2) our incontinence outcome measures were more clearly defined, and (3) we included the $9 \%$ of subjects with preoperative DO, and they excluded preoperative DO. Given these conflicting results, in different directions, we think it is highly unlikely that ODP can predict postoperative incontinence.

Table 3 Preoperative voiding detrusor pressures in urodynamic stress incontinent subjects and postoperative DO

\begin{tabular}{|c|c|c|c|c|c|c|}
\hline & \multicolumn{3}{|c|}{ No preoperative DO $(n=167)$} & \multicolumn{3}{|c|}{ Preoperative DO $(n=20)$} \\
\hline & $\begin{array}{l}\text { No postoperative } \\
\text { DO }(n=152)\end{array}$ & $\begin{array}{l}\text { Postoperative } \\
\text { DO }(n=15)\end{array}$ & $p$ value & $\begin{array}{l}\text { No postoperative } \\
\text { DO }(n=11)\end{array}$ & $\begin{array}{l}\text { Postoperative } \\
\text { DO }(n=9)\end{array}$ & $p$ value \\
\hline ODP & $12.4(9.3)$ & $11.4(7.4)$ & 0.68 & $17.0(11.3)$ & $15.8(8.3)$ & 0.79 \\
\hline$p_{\text {det }} Q_{\max }$ & $19.6(12.0)$ & $19.3(8.1)$ & 0.91 & $21.5(12.7)$ & $22.4(11.7)$ & 0.86 \\
\hline CDP & $16.8(13.6)$ & $19.9(13.9)$ & 0.41 & $16.3(12.5)$ & $21.4(16.3)$ & 0.43 \\
\hline
\end{tabular}

Values are reported as mean in centimeters $\mathrm{H}_{2} \mathrm{O}(\mathrm{SD})$

$O D P$ opening detrusor pressure, $p_{\text {det }} Q_{\max }$ detrusor pressure at maximum flow, $C D P$ closing detrusor pressure, $D O$ detrusor overactivity 
Table 4 Preoperative voiding detrusor pressures in subjects treated for postoperative UUI

\begin{tabular}{lccc}
\hline & Subjects not treated for UUI $(n=225)$ & Subjects treated for UUI $(n=55)$ & $p$ value \\
\hline ODP & $13.1(9.5)$ & $15.3(10.7)$ & 0.13 \\
$p_{\operatorname{det}} Q_{\max }$ & $19.3(11.0)$ & $21.5(12.6)$ & 0.22 \\
CDP & $18.3(16.2)$ & $18.1(14.1)$ & 0.91 \\
\hline
\end{tabular}

Values are reported as mean in centimeters $\mathrm{H}_{2} \mathrm{O}(\mathrm{SD})$

$O D P$ opening detrusor pressure, $p_{\text {det }} Q_{\max }$ detrusor pressure at maximum flow, $C D P$ closing detrusor pressure, $U U I$ urge urinary incontinence

Also, unlike previous investigators who reported that subjects with persistent postoperative DO had ODP values $17 \mathrm{~cm} \mathrm{H}_{2} \mathrm{O}$ higher than subjects without persistent postoperative $\mathrm{DO},[2]$ we found that subjects with persistent postoperative DO had ODP values $1 \mathrm{~cm} \mathrm{H}_{2} \mathrm{O}$ lower than subjects without persistent postoperative DO. Our studies were similar in that both were small (35 and 20 subjects, respectively), but they differed in that our study was Burch and autologous fascial sling surgeries, and their study was TVTs. However, if these preoperative urodynamic variables could reliably predict surgical outcomes, we think they should predict outcomes for both traditional incontinence surgeries and the newer midurethral slings.

In SISTEr, only $9 \%$ of subjects had preoperative DO, but $93 \%$ had a positive response to at least one urge question on MESA [15], therefore, we consider our subjects to have anywhere between a $9 \%$ and $93 \%$ mixed incontinence rate. Because of the ambiguity of the mixed incontinence definition, we decided to concentrate our study on the broader, more generalizable question of preoperative voiding detrusor pressure predictors in all patients undergoing SUI surgery rather than only a small subgroup of subjects with preoperative urodynamic mixed incontinence. Therefore, we also studied 187 subjects without preoperative DO and found similar preoperative voiding detrusor pressures for those with and without postoperative DO.

We chose to evaluate postoperative UUI using the two most unambiguous measures: DO on UDS and treatment for UUI. DO on UDS was the outcome chosen by previous investigators and most likely reflects the most severe UUI patients. Treatment for UUI has a clear definition in our study and arguably is the most clinically relevant overactive bladder condition. We recognize that both of these definitions have limitations. DO on UDS represents events during just a single filling cycle and is not sensitive for the detection of UUI. Treatment for UUI can be influenced by unmeasured variability in clinician and patient preferences with respect to treatment of any UUI that is present. We found no difference in preoperative voiding detrusor pressures for postoperative DO or postoperative treatment for UUI. Given all these results, we think it is highly unlikely that preoperative voiding detrusor pressures can predict postoperative DO and urge incontinence.
The strengths of this study include the large subject population, the multiple sites which improve generalizability, and our careful, standardized urodynamic quality control process. This quality control process excludes poor quality signals. Most urodynamic publications do not perform quality control on their signals and do not evaluate and exclude signals with poor pressure transmission concordance on prevoid cough spikes. A quality control process, especially for pressure-flow studies, which are the most prone to quality challenges, may result in the exclusion of a large number of studies and has the potential to introduce selection bias, but it is unlikely in this study because our postoperative outcome rates were the same in our included and excluded subjects. Therefore, we think insisting on the highest quality, valid and plausible studies strengthens our results and conclusions rather than weakens them by limiting sample size.

In this same study population, other urodynamic predictors were not found to be good predictors of success or failure. [16] It is likely that during preoperative counseling, clinicians will continue to rely on simple clinical factors to predict postoperative outcomes, such as a patient's age, as a predictor of failure and postoperative UUI. [17, 18] Our results suggest no change in this practice.

In conclusion, we did not find that voiding detrusor pressures during preoperative urodynamic studies predict postoperative success, stress-specific success, DO, or treatment of UUI in a large sample of women undergoing Burch or fascial sling procedures for SUI. We do not recommend routine measuring of these urodynamic measures for predictive or prognostic purposes in women undergoing preoperative urodynamics before stress incontinence surgery.

\section{Conflicts of interest None.}

Funding This study was supported by cooperative agreements (U01 DK58225, U01 DK58229, U01 DK58234, U01 DK58231, U01 DK60379, U01 DK60380, U01 DK60393, U01 DK60395, U01 DK60397, and U01 DK60401) with the National Institute of Diabetes and Digestive and Kidney Diseases and by the National Institute of Child Health and Human Development and Office of Research in Women's Health of the National Institutes of Health. 
Open Access This article is distributed under the terms of the Creative Commons Attribution Noncommercial License which permits any noncommercial use, distribution, and reproduction in any medium, provided the original author(s) and source are credited.

\section{References}

1. Digesu GA, Khullar V, Cardozo L, Sethna F, Salvatore S (2004) Preoperative pressure-flow studies: useful variables to predict the outcome of continence surgery. BJU Int 94:1296-1299

2. Panayi DC, Duckett J, Digesu GA, Camarata M, Basu M, Khullar V (2009) Pre-operative opening detrusor pressure is predictive of detrusor overactivity following TVT in patients with pre-operative mixed urinary incontinence. Neurourol Urodyn 28:82-85

3. Tennstedt S (2005) Design of the stress incontinence surgical treatment efficacy trial (SISTEr). Urology 66:1213-1217

4. Herzog AR, Diokno AC, Fultz NH (1989) Urinary incontinence: medical and psychosocial aspects. Annu Rev Gerontol Geriatr 9:74-119

5. Crystle CD, Charme LS, Copeland WE (1971) Q-tip test in stress urinary incontinence. Obstet Gynecol 38:313-315

6. Abrams P, Cardozo L, Fall M, Griffiths D, Rosier P, Ulmsten U et al (2002) The standardisation of terminology of lower urinary tract function: report from the Standardisation Sub-Committee of the International Continence Society. Am J Obstet Gynecol 187:116-126

7. Schafer W, Abrams P, Liao L, Mattiasson A, Pesce F, Spangberg A et al (2002) Good urodynamic practices: uroflowmetry, filling cystometry, and pressure-flow studies. Neurourol Urodyn 21:261274
8. Nager CW, Albo ME, Fitzgerald MP, McDermott SM, Kraus S, Richter HE et al (2007) Process for development of multicenter urodynamic studies. Urology 69:63-67, discussion 67-68

9. Nager CW, Albo ME, Fitzgerald MP, McDermott S, Wruck L, Kraus S et al (2007) Reference urodynamic values for stress incontinent women. Neurourol Urodyn 26:333-340

10. Sullivan J, Lewis P, Howell S, Williams T, Shepherd AM, Abrams P (2003) Quality control in urodynamics: a review of urodynamic traces from one centre. BJU Int 91:201-207

11. Abrams P (2006) Urodynamics. Springer, London, p 75

12. Albo ME, Richter HE, Brubaker L, Norton P, Kraus SR, Zimmern $\mathrm{PE}$ et al (2007) Burch colposuspension versus fascial sling to reduce urinary stress incontinence. N Engl J Med 356: 2143-2155

13. Bump RC, Mattiasson A, Bo K, Brubaker LP, DeLancey JO, Klarskov $P$ et al (1996) The standardization of terminology of female pelvic organ prolapse and pelvic floor dysfunction. Am J Obstet Gynecol 175:10-17

14. Hintze J. (2008) PASS 2008. NCSS, LLC. Kaysville, Utah. www. ncss.com

15. Brubaker L, Stoddard A, Richter H, Zimmern P, Moalli P, Kraus SR et al (2009) Mixed incontinence: comparing definitions in women having stress incontinence surgery. Neurourol Urodyn 28:268-273

16. Nager CW, FitzGerald M, Kraus SR, Chai TC, Zyczynski H, Sirls $\mathrm{L}$ et al (2008) Urodynamic measures do not predict stress continence outcomes after surgery for stress urinary incontinence in selected women. J Urol 179:1470-1474

17. Richter HE, Goode PS, Brubaker L, Zyczynski H, Stoddard AM, Dandreo KJ et al (2008) Two-year outcomes after surgery for stress urinary incontinence in older compared with younger women. Obstet Gynecol 112:621-629

18. Richter HE, Diokno A, Kenton K, Norton P, Albo M, Kraus S et al (2008) Predictors of treatment failure 24 months after surgery for stress urinary incontinence. J Urol 179:1024-1030 